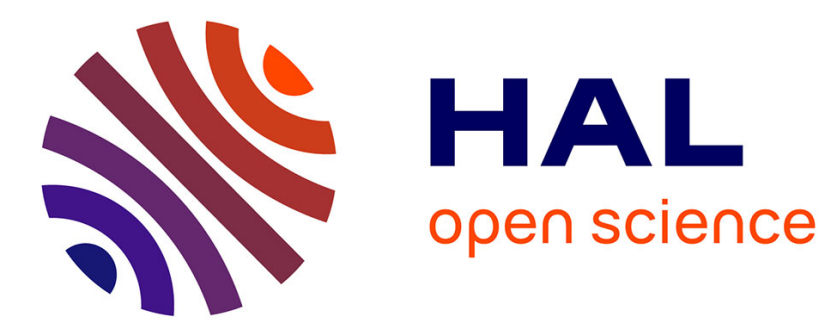

\title{
Three-dimensional study of multiple transitions for natural convection in horizontal annuli
}

\author{
G. Petrone, Eric Chénier, Guy Lauriat
}

\section{To cite this version:}

G. Petrone, Eric Chénier, Guy Lauriat. Three-dimensional study of multiple transitions for natural convection in horizontal annuli. International Journal of Heat and Mass Transfer, 2006, 49 (7-8), pp.1231-1241. 10.1016/j.ijheatmasstransfer.2005.10.017 . hal-00693764

\section{HAL Id: hal-00693764 \\ https://hal.science/hal-00693764}

Submitted on 3 Jun 2017

HAL is a multi-disciplinary open access archive for the deposit and dissemination of scientific research documents, whether they are published or not. The documents may come from teaching and research institutions in France or abroad, or from public or private research centers.
L'archive ouverte pluridisciplinaire HAL, est destinée au dépôt et à la diffusion de documents scientifiques de niveau recherche, publiés ou non, émanant des établissements d'enseignement et de recherche français ou étrangers, des laboratoires publics ou privés. 


\title{
Three-dimensional study of multiple
}

\section{transitions for natural convection in horizontal}

\section{annuli}

\author{
G. Petrone, E. Chénier and G. Lauriat* \\ University of Marne-la-Vallée, LETEM \\ bât. Lavoisier, Champs-sur-Marne \\ F-77454 Marne-la-Vallée cedex 2
}

\begin{abstract}
Natural convection in horizontal differentially heated annuli is numerically investigated both by linear stability analysis and by solving the three-dimensional flow equations for a radius ratio $R=1.7$. The governing equations were solved numerically by using a finite volume method. The stability of the basic crescent-shaped flows to three-dimensional disturbances is investigated. The existence of a stability region corresponding to a reversal from 3D- to $2 \mathrm{D}$-flows is predicted for the first time. Three-dimensional computations show that multiple solutions are possible in the new stability region according to the initial conditions. Computations performed at supercritical Rayleigh numbers for the oscillatory régime elucidated the influence of the annulus length. Conflicting results reported in the archival literature are clarified.
\end{abstract}

\footnotetext{
* Corresponding author: Tel.: +33-1-60-95-72-69; fax: +33-1-60-95-72-94

Email addresses: chenier@univ-mlv.fr (E. Chénier), lauriat@univ-mlv.fr
}

Preprint submitted to Elsevier Science

25 October 2005 


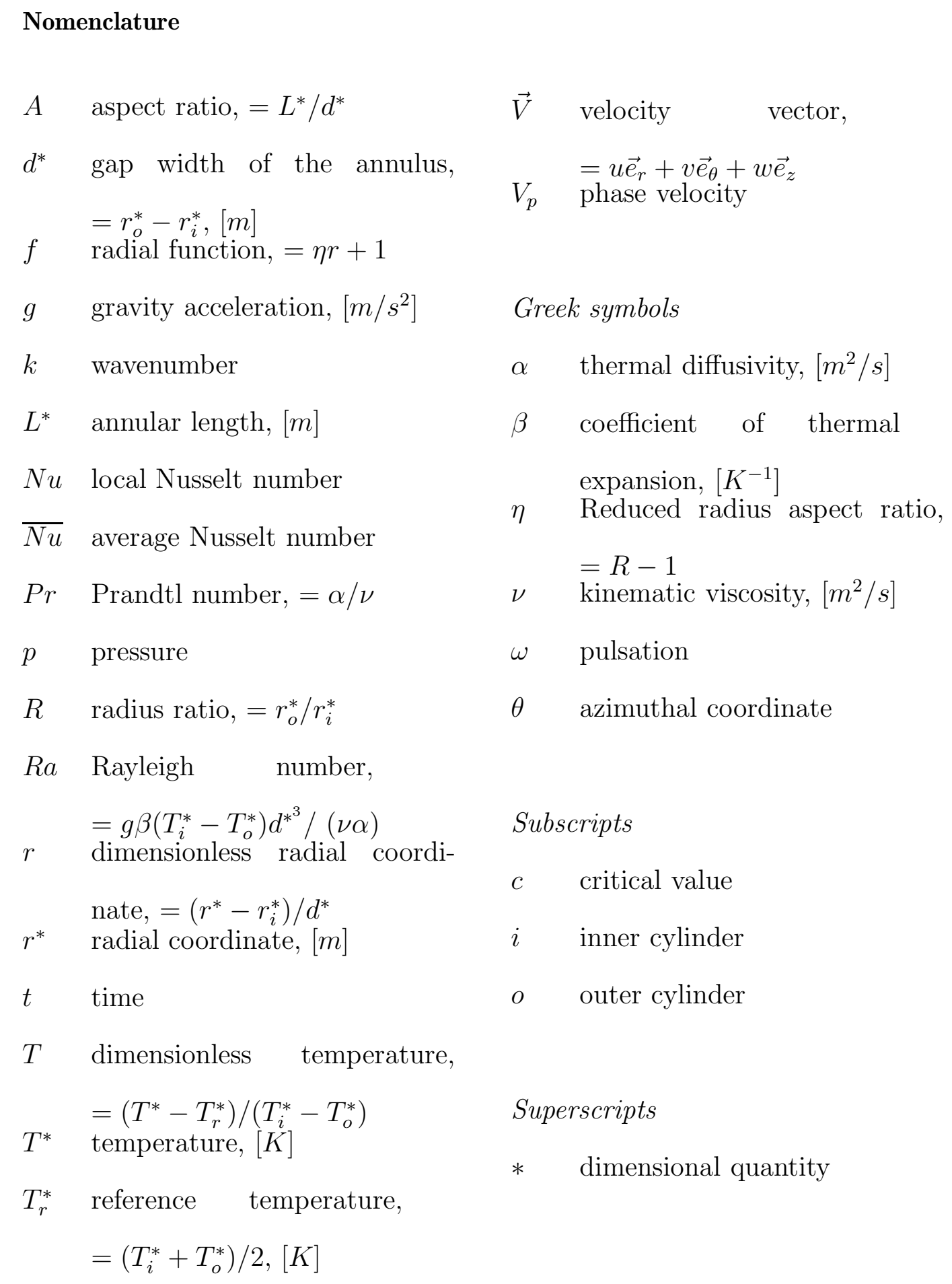

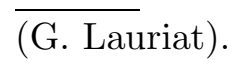




\section{Introduction}

Natural convection in square or rectangular cavities, with horizontal or vertical isothermal walls, has been extensively studied and a large variety of flow patterns has been discussed in the archival literature. When studying natural convection in horizontal differentially heated annulus, it can be considered that both orientations of the thermal gradients are simultaneously applied, in the upper/lower regions and lateral regions, respectively. Thus, a rich diversity of flow structures is encountered according to the radius ratio of the cylinders, $R$.

The two-dimensional air flow patterns were classified as a function of the radius ratio in a recent paper by Petrone et al. [1]: in addition to the usual couple of counter-rotating crescent-shaped cells, located within each transversal section of the annulus, various multi-cellular flow patterns were predicted. A map of 2D-stable flow patterns, based on two-dimensional linear stability calculations, was established in the $(R, R a)$-parameter space. This map clearly highlights the regions where multiple solutions may exist.

To our best knowledge, the first three-dimensional numerical investigations were published around the mid-eighties by Tanaka et al. [2] and Rao et al. [3] for high Prandtl number fluids $\left(\operatorname{Pr}=5 \cdot 10^{3}\right)$. For $R=2, A=4$ and $1.25 \cdot 10^{3} \leq R a \leq 1.25 \cdot 10^{4}$, the flow was shown weakly affected by the end walls: the fluid particle trajectories lie essentially in the cross-section of the annulus, as for two-dimensional solutions, with a very small axial dependency. This flow pattern was classified as helical. For a smaller radius ratio $(R=1.428), A=6.66$ and $1500<R a<8100$, the flow results from a combi- 
nation of two origins of fluid motions and is named as spiral flow. In the lower region of the annulus, the helical structure persists whereas counter-rotating transverse rolls (with respect to the annulus axis) develop in the upper part of the annular space. These rolls correspond to a thermal instability of the helical flow and they occur between two almost parallel plates differentially heated, as in the Rayleigh-Bénard configuration.

Using air as the working fluid in a large-gap annulus of small aspect ratio ( $R=2.64, A=1.625)$, Fusegi and Farouk [4] showed the effect of the end walls on fluid motions for $10^{3} \leq R a \leq 10^{4}$. Vafai and Ettefagh [5] studied the transient flows and the influence of the aspect ratio $(A \geq 6.5)$ for $R=2.6$ and $116 \leq R a \leq 10^{4}$. The flow in the core region of these annuli remains nearly two-dimensional, the extent of the core region decreasing however with $R a$. For narrow gap annuli $(R=1.2), 6 \leq A \leq 50$ and $R a \approx 3 \cdot 10^{3}$, Cadiou [6] explained the onset of the steady three-dimensional spiral flow by the propagation of disturbances from the end walls towards the core region of the annulus. The elapsed time necessary to destabilize the two-dimensional pattern in the mid-axial plane was assumed almost proportional to the aspect ratio of the annulus. Recently, Dyko et al. [7] confirmed the occurrence of the spiral flow structure for moderate-gap annuli $(1.2 \leq R \leq 1.6)$, while the two-dimensional counter-rotating crescent-shaped cells were observed in an annulus of larger radius ratio $(R=2.2)$. In this latter case, the viscous shear forces produce transverse cells near each of the end walls. For narrow-gap annuli $(R \leq 1.2)$, Dyko and Vafai $[8,9]$ found complex flow patterns produced by the combination of upper transverse rolls with two-dimensional multi-cellular flows. These flow patterns are similar to those discussed in Petrone et al. [1]. 
Most of these numerical results are in quite good agreement with the map established by Powe et al. [10] and based upon a number of experiments. For radius ratios larger than $R=1.71$ the flow structure is mainly twodimensional, at first steady and then oscillatory above a critical Rayleigh number much higher than the Rayleigh numbers considered in all the threedimensional numerical simulations published so far. For moderate radius ratios $(1.24 \leq R \leq 1.71)$, a thermal instability occurs in the upper region of the annulus, and a three-dimensional spiral flow emerges at supercritical Rayleigh numbers. For small radius ratios $(R<1.24)$, the basic unicellular steady régime (crescent-shaped cells) changes to a steady multi-cellular flow régime. For $1.3 \leq R \leq 6.3$, Grigull and Hauf [11] experimentally observed both three-dimensional spiral and two-dimensional crescent-shaped flows in the range $1680 \leq R a \leq 21,000$. For $R a<1680$ or $R a>21,000$, the flows develop essentially in the two-dimensional cross-section of the annulus.

The linear stability analysis, performed by Choi and Kim [12] for moderate radius ratios $(1.24 \leq R<1.71)$, showed the onset of disturbances in the transverse section of the annulus above critical Rayleigh numbers close to 2000. Theses perturbations look like the transverse rolls observed as well in experimental works as in numerical simulations. However, this linear stability analysis does not predict the two-dimensional flows observed above $R a=$ $2.1 \cdot 10^{4}$ by Grigull and Hauf [11]. Moreover for $1.71 \leq R \leq 2$, the computed thresholds are about one order of magnitude smaller than the values reported by Powe et al. [10] on their stability map (see Fig.6 in [12]).

In this paper, we examine natural convection in air-filled annuli. Most of the discussion is focused on the case $R=1.7$. The stability of the basic twodimensional, crescent-shaped flows to small three-dimensional disturbances 
was investigated first. The existence of a stability region corresponding to a reverse transition from 3D- to 2D-flows is predicted for the first time. Having determined the critical Rayleigh numbers, three-dimensional computations were performed for annuli with solid end walls. The governing equations for the primitive variables were solved numerically in the transient form by using a finite volume method with second order time and space discretizations. The numerical implementation was adapted for a vectorial computer, with efficient solvers for the Helmholtz and Poisson problems. It is shown that multiple solutions are possible in the new stability region according to the initial conditions. Computations carried-out for supercritical Rayleigh numbers in the oscillatory régime elucidated the influence of the annulus length and the results are compared to the experiments conducted by Grigull and Hauf [11].

\section{Physical model}

\subsection{Governing equations}

A schematic of the horizontal air-filled annulus considered for the purpose of the present analysis is shown in Fig. 1. The annulus of length $L^{*}$ is formed by two co-axial cylinders of radii $r_{i}^{*}<r_{o}^{*}$, held at uniform temperatures $T^{*}\left(r_{i}\right)=$ $T_{i}^{*}>T^{*}\left(r_{o}\right)=T_{o}^{*}$ and bounded by two impermeable and adiabatic plates. The flow is assumed to be laminar and incompressible with constant physical properties except the density in formulating the buoyancy effect. By scaling the axial coordinate by the annulus gap $d^{*}=r_{o}^{*}-r_{i}^{*}$, the velocity components by the thermal diffusivity velocity $\alpha / d^{*}$, by introducing the dimensionless

temperature difference $T=\left(T^{*}-T_{r}^{*}\right) /\left(T_{i}^{*}-T_{o}^{*}\right)$ where $T_{r}^{*}=\left(T_{i}^{*}+T_{o}^{*}\right) / 2$ 
is the reference temperature, and the dimensionless radial coordinate $r=$ $\left(r^{*}-r_{i}^{*}\right) / d^{*}$, then the dimensionless governing equations write in cylindrical coordinates $(r, \theta, z)$ :

$$
\begin{aligned}
& \overline{\widetilde{\nabla}} \cdot \vec{V}=0 \\
& \frac{\partial}{\partial t}(f u)+\overline{\widetilde{\nabla}} \cdot(u \vec{V})-\eta v^{2}=-f \frac{\partial p}{\partial r}-\operatorname{RaPrf} T \cos \theta+\operatorname{Pr}\left(\widetilde{\nabla}^{2} u-\frac{2 \eta^{2}}{f} \frac{\partial v}{\partial \theta}-\frac{\eta^{2} u}{f}\right) \\
& \frac{\partial}{\partial t}(f v)+\overline{\widetilde{\nabla}} \cdot(v \vec{V})+\eta u v=-\eta \frac{\partial p}{\partial \theta}+\operatorname{RaPrf} T \sin \theta+\operatorname{Pr}\left(\widetilde{\nabla}^{2} v+\frac{2 \eta^{2}}{f} \frac{\partial u}{\partial \theta}-\frac{\eta^{2} v}{f}\right) \\
& \frac{\partial}{\partial t}(f w)+\overline{\widetilde{\nabla}} \cdot(w \vec{V})=-f \frac{\partial p}{\partial z}+\operatorname{Pr} \widetilde{\nabla}^{2} w \\
& \frac{\partial}{\partial t}(f T)+\overline{\widetilde{\nabla}} \cdot(T \vec{V})=\widetilde{\nabla}^{2} T
\end{aligned}
$$

where $\eta=r_{o}^{*} / r_{i}^{*}-1$ and $f=\eta r+1$. The spatial operators are defined as follows:

$$
\overline{\widetilde{\nabla}} \cdot(X \vec{V})=\frac{\partial(f X u)}{\partial r}+\frac{\partial(\eta X v)}{\partial \theta}+\frac{\partial(f X w)}{\partial z}
$$

and

$$
\widetilde{\nabla}^{2} X=\frac{\partial}{\partial r}\left(f \frac{\partial X}{\partial r}\right)+\frac{\partial}{\partial \theta}\left(\frac{\eta^{2}}{f} \frac{\partial X}{\partial \theta}\right)+\frac{\partial}{\partial z}\left(f \frac{\partial X}{\partial z}\right)
$$

where $X(r, \theta, z)$ is a scalar quantity.

The problem is then characterized by four dimensionless parameters: the Prandtl number, $\operatorname{Pr}=\nu / \alpha$, the Rayleigh number based on the annulus thickness, $R a=g \beta\left(T_{i}^{*}-T_{o}^{*}\right) d^{*^{3}} /(\nu \alpha)$, the radius ratio, $R=r_{o}^{*} / r_{i}^{*}$, and the axial aspect ratio, $A=L^{*} / d^{*}$. 
The corresponding boundary conditions are

$$
\begin{aligned}
& u=v=w=0, T=+0,5 \quad \text { at } \quad r=0 \\
& u=v=w=0, T=-0,5 \text { at } \quad r=1 \\
& u=v=w=0, \frac{\partial T}{\partial z}=0 \text { at } z=0 \text { and } z=A
\end{aligned}
$$

Taking into account the symmetry of both the flows observed in the experiments and the dominant disturbances computed by our stability analyses, only the half annulus is considered for the three-dimensional simulations with symmetry conditions applied at $\theta=0$ and $\pi$. These conditions imply:

$$
\frac{\partial u}{\partial \theta}=0, v=0, \frac{\partial w}{\partial \theta}=0, \frac{\partial T}{\partial \theta}=0, \quad \text { at } \quad \theta=0 \text { and } \theta=\pi
$$

\subsection{Heat transfer}

The local Nusselt number at any axial location on the cylinder surfaces was calculated by

$$
N u(\theta, z)=-\left.\ln (R)\left(r_{c}+\frac{1}{\eta}\right) \frac{\partial T}{\partial r}\right|_{r_{c}}
$$

where $r_{c}$ stands for $r_{i}=0$ or $r_{o}=1$.

The overall mean Nusselt number was evaluated from the spatial average of the total heat transfer as:

$$
\overline{N u}_{3 D}=\frac{1}{\pi A} \int_{0}^{A} \int_{0}^{\pi} N u(\theta, z) d \theta d z
$$


We defined also the mean Nusselt number at the mid-axial plane as:

$$
\overline{N u}_{c}=\frac{1}{\pi} \int_{0}^{\pi} N u\left(\theta, \frac{A}{2}\right) d \theta
$$

The difference between the inner and outer cylinder mean Nusselt numbers, $\overline{N u}_{i}$ and $\overline{N u}_{o}$, respectively, indicates the importance of 3D-effects in the core region of the annulus since $\overline{N u}_{i}=\overline{N u}_{o}=\overline{N u}_{2 D}$ at steady state conditions.

\section{Numerical methods}

\subsection{Grid distributions}

The governing equations with the boundary conditions were solved numerically in the primitive variable formulation by using a finite volume method on a staggered but structured grid. For a half-annulus, the mesh defined by $\left[r_{i}, r_{i+1}\right] \times\left[\theta_{j}, \theta_{j+1}\right] \times\left[z_{k}, z_{k+1}\right]$ was built up as:

$$
\begin{aligned}
& r_{i}=\frac{\tanh \left(c_{r}\left(2 \frac{i-1}{N_{r}}-1\right)\right)+\tanh \left(c_{r}\right)}{2 \tanh \left(c_{r}\right)}, \quad i=1, N_{r}+1 \\
& \theta_{j}=\pi \frac{\exp \left(\pi c_{\theta} \frac{j-1}{N_{\theta_{1}}}\right)-1}{\exp \left(\pi c_{\theta}\right)-1}, \quad j=1, N_{\theta_{1}}+1 \\
& z_{k}=A \frac{\tanh \left[c_{z}\left(2 \frac{k-1}{N_{z}}-1\right)\right]+\tanh \left(c_{z}\right)}{2 \tanh \left(c_{z}\right)}, \quad k=1, N_{z}+1
\end{aligned}
$$

For the computations of the basic two-dimensional steady states and the dominant disturbances, the full angular domain was considered:

$$
\theta_{j}= \begin{cases}\pi \frac{\exp \left(2 \pi c_{\theta} \frac{j-1}{N_{\theta_{2}}}\right)-1}{\exp \left(\pi c_{\theta}\right)-1}, & j=1, \frac{N_{\theta_{2}}}{2}+1 \\ 2 \pi-\theta_{N_{\theta_{2}}+2-j}, & j=\frac{N_{\theta_{2}}}{2}+1, N_{\theta_{2}}+1\end{cases}
$$


The variables $N_{r}, N_{z}, N_{\theta_{1}}$ are any integers and $N_{\theta_{2}}$ is an even integer. In order to adjust the grid distribution at the upper part of the annulus (near $\theta=\pi)$ and in the wall regions, three strictly negative real parameters $\left(c_{r}, c_{\theta}\right.$ and $c_{z}$ ) were introduced. If one of these three parameters was zero, the grid distribution was uniform in the corresponding direction.

\subsection{Three-dimensional temporal scheme}

The transport terms of the momentum and energy equations were discretized by using a second-order centered scheme. The time integration was performed by an implicit second-order Euler scheme and an Adams-Bashforth extrapolation for the diffusion and transport terms, respectively. The time splitting method [13] was used to uncouple velocity and pressure fields. The Helmholtz equations, stemming from the Euler scheme, were solved by an incremental factorization method which consists in rewriting first the transport equations by introducing the time increment of the variable instead of the variable itself. The 3D-Helmholtz operator is then transformed into a product of three elementary 1D-Helmholtz operators, each of them acting in one space direction (a more detailed explanation is given in the Appendix A.1). The pressure correction equation $(1 / f) \widetilde{\nabla}^{2} \phi^{(n)}=S^{(n)}$, resulting from the use of the Goda scheme [13], was solved by computing the solution $\phi^{(n)}=p^{(n+1)}-p^{(n)}$ into the eigenspaces of the partial second-order derivatives $\left(\partial^{2}\right) /\left(\partial \theta^{2}\right)$ and $\left(\partial^{2}\right) /\left(\partial z^{2}\right)$. This procedure, similar to the diagonalization technique often used with spectral or pseudospectral methods [14], is well suited to the present problem. It is described in Appendix A.2.

The second-order space and time discretizations are successfully checked by 
introducing volumetric source terms in the governing equations (Appendix B).

\subsection{Stability analysis}

The procedure used to conduct the linear stability analysis was described in Petrone et al. [1] for two-dimensional perturbations. Briefly, it consists first in the calculation of a two-dimensional basic flow by solving the steady conservative equations by using the Newton-Raphson method. The most unstable perturbation, developed in Fourier series in the $z$-direction, is then obtained with the Arnoldi method for each wavenumber $k$.

For a given wavenumber, the threshold parameter $R a_{c}$ is iteratively evaluated by using the Lagrange method. It consists in solving the implicit non-linear equation $\lambda\left(R a_{c}\right)=0$ as follows:

Let us assume that two Rayleigh number values $R a^{(0)}$ and $R a^{(1)}$ were determined so that the growth rates $\lambda^{(0)}$ and $\lambda^{(1)}$ of the dominant perturbation satisfy $\lambda^{(0)} \times \lambda^{(1)}<0$. Starting from $i=0$, the algorithm converges toward $R a_{c}$ :

(1) $R a^{(i+2)}=\frac{R a^{(i)} \times \lambda^{(i+1)}+R a^{(i+1)} \times \lambda^{(i)}}{\lambda^{(i)}+\lambda^{(i+1)}}$

(2) The basic flow and then the growth rate $\lambda^{(i+2)}$ are computed at $R a^{(i+2)}$;

(3) - If $\lambda^{(i+2)}$ is small enough then $R a_{c} \approx R a^{(i+2)}$, stop.

- If $\lambda^{(i+2)} \times \lambda^{(i)}<0$, then $R a^{(i+3)}=R a^{(i)}$ else $R a^{(i+3)}=R a^{(i+1)}$

(4) $i=i+2$, go to step (1).

For long enough annuli, the critical Rayleigh number $R a_{c_{\infty}}=\min _{k}\left(R a_{c}\right)$ (respectively $R a_{c_{\infty}}=\max _{k}\left(R a_{c}\right)$ ) for a destabilizing (respectively stabilizing) transition is computed by a quadratic interpolation based on the nearest 
threshold values $R a_{c}(k)$.

\section{Results}

Stability analysis and simulations were conducted for air $(\operatorname{Pr}=0.7)$ with the radius ratio set to $R=1.7$, except in the comparison section.

\subsection{Comparisons with the three-dimensional results of Dyko et al. [7]}

The simulations were first performed to match with results recently published by Dyko et al. [7]. For comparison purposes, isothermal patterns and velocity vectors were plotted in the vertical $(r, z)$ upper section of the annulus.

The computations were carried out for $A=6.66, R=1.6$, at $R a=10^{3}$ and $R a=2 \cdot 10^{3}$ with a grid resolution defined by $\left(N_{r}, N_{\theta}, N_{z}\right)=(40,120,280)$ and $\left(c_{r}, c_{\theta}, c_{z}\right)=(0,-0.75,-0.25)$. Figures $2 \mathrm{a}-\mathrm{b}$ show the onset of upper transverse rolls produced by thermal instabilities. Despite a convection of the thermal field slightly more pronounced in Fig. 2b than in Dyko et al. [7], the isotherms agree qualitatively well.

For $R=2.2$ and $A=8.33$, simulations were performed at $R a=10^{3}, 4$. $10^{3}$ and $10^{4}$. The mesh was $\left(N_{r}, N_{\theta}, N_{z}\right)=(40,120,600)$ and $\left(c_{r}, c_{\theta}, c_{z}\right)=$ $(0,-0.75,-0.25)$. In contrast with the previous solutions for a smaller radius ratio, transverse rolls do not develop in the upper region of the annulus (Figs. 3a-c) while increasing $R a$ enhances the convective transfer. As it can be seen, the isotherms are almost horizontal except near the end walls where the no-slip conditions are at the origin of rolls confined close to the boundaries. Although the isotherms are graphically similar with [7], some small differences 
are noticeable close to the end walls. The isotherms of Fig. 3b-c seems to be more distorted for $R a=4 \cdot 10^{3}$ and less affected by the convective cells at $R a=10^{4}$. The streamlines and isotherms, drawn in the vertical mid crosssection at $z=A / 2$, are also in good agreement with those presented in [7]. At last, the angular averages of the Nusselt numbers evaluated at the walls for $z=A / 2$ differ from the results of [7] by less than $3.5 \%$.

\subsection{Stability analysis}

At small enough Rayleigh numbers (i.e. $R a<\cong 2000$ ), the flow is mainly two-dimensional, the three-dimensional effects being noticeable close to the end walls only. Therefore, the flow is composed of the well-studied couple of counter-rotating crescent-shaped cells, symmetrically located in each of the $\pi$ -annular sections. The linear stability analysis may be thus performed for twodimensional basic flows, with the axial disturbances expressed in Fourier series in the $z$-direction. The effects of finite length annuli with no-slip conditions on the end walls are thus not taking into account in the results discussed in the present section.

A previous work showed that the $2 \mathrm{D}$ crescent-shaped flows were stable with respect to two-dimensional disturbances up to $R a<10,000$ [1]. The present study indicates that the basic flow is in fact destabilized at a smaller Rayleigh number value, $R a_{c_{1}}=2045$, by the onset of a $3 \mathrm{D}$ perturbation with a wavenumber close to $\pi\left(k_{c_{1}}=3.07\right)$. The superimposition of basic flow and perturbation gives rise to upper transverse rolls, named as the spiral flow. These results are in excellent agreement with the linear stability analysis by Choi and Kim [12]. Increasing $R a$ leads to a reverse transition to stable two-dimensional flows. 
The transition occurs above a critical value $R a_{c_{2}}=7612$ with a wavenumber

$k_{c_{2}}=4.07$. The destabilizing and stabilizing transitions are driven by the same steady perturbation mode, respectively amplified or damped when increasing $R a$.

The crescent-shaped flow is then linearly stable until the onset of an oscillatory disturbance occurring at $R a_{c_{3}}=19,947$. This mode is three-dimensional with a wavenumber close to $\pi$ again $\left(k_{c_{3}}=3.2\right)$ and a dimensionless pulsation $\omega_{c_{3}}=50$.

It is worth noticing that

(1) the critical value $R a_{c_{3}}$ is about ten times larger than the first instability threshold $R a_{c_{1}}$;

(2) the two-dimensional linear stability region $R a_{c_{2}}<R a<R a_{c_{3}}$ is about twice more extended than the instability region defined by $R a_{c_{1}}<R a<$ $R a_{c_{2}}$

The discrepancy between the linear stability results of Choi and Kim [12], indicating that the flow is three-dimensional above $R a c_{1}$, and experimental results $[10,11]$, showing that $2 \mathrm{D}$ flows exist for larger Rayleigh numbers, is thus clarified for the first time.

\subsection{Three-dimensional simulations}

Three-dimensional simulations were performed in order to study the flows at Rayleigh number values lying in the stability and instability regions predicted by the stability analysis conducted for annuli of infinite length.

Using the symmetry properties of both the basic flows and of the perturba- 
tions, computations were restricted to half-annular spaces with symmetrical azimuthal conditions for $\theta=0$ and $\pi$. Except for the last simulation, where the grid parameters and the annulus aspect ratio will be specified later on, the mesh used for all the other computations was defined by $\left(N_{r}, N_{\theta}, N_{z}\right)=$ $(60,120,360)$ with $\left(c_{r}, c_{\theta}, c_{z}\right)=(0,-0.5,0)$ and the axial aspect ratio was kept constant with $A=6$. A finer grid, $\left(N_{r}, N_{\theta}, N_{z}\right)=(90,180,540)$, was also used but not any noticeable differences in the flow quantities was exemplified. The time step was lying in the range $10^{-3} \leq \Delta t \leq 2.5 \cdot 10^{-4}$.

\subsubsection{First instability region}

A simulation was performed for $R a=6000$, namely in the first instability region $] R a_{c_{1}}, R a_{c_{2}}$. In agreement with the results of Dyko et al. [7], the flow was found steady. Fluid particles, injected in the vertical $(r, z)$ upper section of the annulus, follow first a spiral motion in the upper part of the cavity and are then ejected in the core region in which a helical motion is observed (Fig. 4). This thermal instability of the crescent-shaped flow gives rise to transverse convective rolls that strongly modify the isothermal pattern in the axial direction (Fig. 5). In contrast to the flow pattern shown in Fig. $2 \mathrm{~b}(R a=$ 2000, $A=6.66$ and $R=1.6$ ), which was symmetric with respect to the vertical mid-plane, the present flow possesses an odd number of transverse cells ( 7 cells) and, consequently, the reflection symmetry is broken. This symmetry breaking was also predicted by Dyko and Vafai [9] for narrow annuli $R<1.2$. 


\subsubsection{Stability region}

Three-dimensional stability analyses of two-dimensional flows have shown that the purely two-dimensional solution is linearly stable at $R a=10^{4}$. In order to see what can be observed in real three-dimensional situations, two threedimensional simulations were performed by using different initial conditions.

First, by starting the computations with a quiescent fluid at uniform temperature $T=0$ as initial conditions, the flow remains steady and mainly two-dimensional in the core region of the annulus. Figure 6a shows that the isotherms plotted in the upper vertical $(r, z)$-plane are almost independent of the axial coordinate, except near the end walls where transverse cells form because of the viscous shear associated with the no-slip boundary condition. However, these two cells do not affect the almost two-dimensional flow, which remains stable within the core region. The relative difference between the inner and outer cylinder mean Nusselt numbers at the mid-axial plane $z=A / 2, \overline{N u}_{i}=1.699$ and $\overline{N u}_{o}=1.732$, respectively, is only $2 \%$. In addition, the Nusselt number computed from a two-dimensional simulation, for which $\overline{N u}_{2 D}=\overline{N u}_{i}=\overline{N u}_{o}=1.715$, and the overall Nusselt number calculated by

the three-dimensional model, $\overline{N u}_{3 D}=1.691$, differ from $1.40 \%$. Therefore, the three-dimensionality caused by the end wall cells has a weak effect on the heat transfer rate, and the flow may be assumed as two-dimensional, in agreement with the predictions of the linear stability analysis performed for an annulus of infinite length.

The second simulation was carried out by using the developed three-dimensional flow computed for $R a=6000$ as an initial condition. Figure 5 shows that the initial number of transverse rolls is $n=7$. The converged solution is steady and fully three-dimensional, but the number of tranverse rolls is reduced to 
$n=6$, as indicated in Fig. 6b. However, the reflection symmetry with respect to the vertical mid cross-section is not satisfied even with this even number of transverse rolls. Such a symmetry breaking, not usual for an even number of transverse rolls, is caused by different stretchings of the end wall cells. The relative difference between the inner and outer cylinder mean Nusselt numbers at the mid-axial plane is now approximately equal to $40 \%\left(\overline{N u}_{i}=1.612, \overline{N u}_{o}\right.$ $=2.253$ ), indicating the importance of the heat transfer in the axial direction. However, the difference between $\overline{N u}_{2 D}=1.715$ and $\overline{N u}_{3 D}=1.788$ is about $4 \%$ only. It can be thus concluded that the heat transfer rate may be evaluated with a reasonable accuracy from results of $2 \mathrm{D}$-simulations. At last, it is worth noting that a similar three-dimensional flow pattern was observed in the experimental work by Grigull and Hauf [11] for neighbouring parameters, namely $R=1.64$ and $R a=13,160$. To explain their result, it is suggested that the increase in the temperature difference applied between the cylinder surfaces required to reach $R a=13,160$ was slow, so that the three-dimensional pattern achieved in the instability region was preserved.

It can thus be concluded that the flow converges towards different solutions according to the initial condition for $R a_{c_{2}}<R a<R a_{c_{3}}$. This multiplicity does not contradict the linear stability analysis, which only gives a sufficient criterion for the amplification of disturbances of infinitesimal amplitudes. To complete this work, an energetic stability analysis could be performed to confirm if the two-dimensional flow is indeed unstable with respect to finite amplitude disturbances. 


\subsubsection{Second instability region}

The last simulations were carried out in the second instability region, for $R a=22,000>R a_{c 3}$ and for various axial aspect ratios.

For $R=1.7$ and $A=6$, the flow converges towards a steady flow, the structure of which being mainly two-dimensional. Also, the $\overline{N u}$-values show that axial heat transfer is almost negligible since $\left(\overline{N u}_{o}-\overline{N u}_{i}\right) / \overline{N u}_{i}=0.23 \%$ $\left(\overline{N u}_{i}=2.1347\right)$ while the relative difference between $\overline{N u}_{2 D}=2.1413$ and $\overline{N u}_{3 D}=2.1108$ is about $1.44 \%$. On one hand, this flow pattern is quite surprising because of the linear stability predictions. On the other hand, this result agrees well with the two-dimensional flow observed experimentally by Grigull and Hauf [11] for $R a>21,000$ and $1.3 \leq R \leq 6.3$. By assuming that the too small aspect ratio could prevent the amplification of 3D-oscillatory disturbances, a new computation was carried-out for a longer annulus, i.e. $A=20$.

For $A=20$, the grid parameter was $\left(N_{r}, N_{\theta}, N_{z}\right)=(40,80,810)$ and $\left(c_{r}, c_{\theta}, c_{z}\right)=$ $(0,-0.25,0)$. The flow obtained was then oscillatory with a pulsation $\omega \approx 50$, very close to $\omega_{c_{3}}$ which is the pulsation predicted by the linear stability analysis at the threshold $R a_{c 3}$. Nevertheless, the flow seems to stay essentially two-dimensional (Fig. 7) with the largest axial components of velocity located near the end walls. For example, the streamfunctions, illustrated by the fluid particle tracking in the core region and at a fixed time, look like those of two-dimensional patterns. However, a careful inspection of the axial component of the velocity in the upper vertical $(r, z)$-plane shows a weak spatial oscillatory behavior. Figure 8 displays that the number of oscillations in the axial direction is approximatively $n=11$. For the aspect ratio $A=20$, the approximate wavenumber which can be deduced is $2 \pi n / A$, namely $k=3.45$. 
The difference between this wavenumber and the wavenumber of the marginal disturbance $\left(k_{c_{3}}=3.2\right)$ is thus small. One can deduce that the spatial and temporal properties of the three-dimensional flow are in good agreement with the three-dimensional stability analysis. To improve the understanding of this complex flow, a spatio-temporal diagram was established for temperature, in the upper vertical $(r, z)$-plane $(\theta=\pi)$ near the cold cylinder $(r=0.9)$. Such a diagram is obtained by plotting the temperature variation $\Delta T$, defined by the difference between its instantaneous value and its time-averaged value, as a function of the axial coordinate. Figure 9 illustrates the crests and holes resulting from opposite propagating waves, from the core region towards the end walls. The interactions of these waves produce the stationary interferogram pattern shown in Fig. 9, with the checkerboard patterns located in the core region of the annulus. The phase velocity of the propagating waves is approximately $V_{p} \approx 13.7$. It is in agreement with the perturbation analysis, $\omega_{c_{3}} / k_{c_{3}}=15.9$. Considering the weakness of the axial component of the velocity within the core region, we suggest to classify this flow as mainly twodimensional.

In conclusion, these simulations for two very separate aspect ratios have shown that the flows are characterized by 2D-patterns in the core region of the annulus. These results agree with the experimental observations by Grigull and Hauf [11] for $R a>21,000$ and $1.3 \leq R \leq 6.3$, at least near the instability threshold. 


\section{Conclusion}

The present work examined natural convection of air between two concentric horizontal cylinders differentially heated. Most of the computations were performed for the radius ratio $R=1.7$. The investigation encompassed both linear stability analyses and complementary numerical solutions.

Three-dimensional linear stability analysis of two-dimensional numerical solutions showed that the first critical Rayleigh number above which the flow is unstable and three-dimensional, $R a_{c_{1}}$, agrees with previously published results. The new findings presented in this paper consist in establishing that two other transitions occur. The first transition (at $R a_{c_{2}}$ ) corresponds to a reverse transition to $2 \mathrm{D}$-solutions whereas the second transition (at $R a_{c_{3}}$ ) is characterized by transient amplifications of disturbances. The critical Rayleigh number for the onset of unsteady three-dimensional flow, $R a_{c_{3}}$, is one order of magnitude higher than $R a_{c_{1}}$.

Three-dimensional simulations were conducted for the different states. In agreement with previous studies, the spiral flow engendered by the amplification of thermal instabilities in the upper part of the annulus was predicted for $R a_{c_{1}}<R a<R a_{c_{2}}$. Multiple solutions are possible in the new stability region $\left(R a_{c_{2}}<R a<R a_{c_{3}}\right)$ highlighted by the linear stability analysis. They are either two- or three-dimensional according to the initial conditions. Simulations performed for $R a=22,000>R a_{c_{3}}$ have shown that the results depend on the axial aspect ratio of the annulus. For an annulus of to small length, the onset of the oscillatory disturbances, predicted by the stability study, is delayed and a steady flow is obtained. For long enough annuli, the flow is oscillatory and the wavenumber and pulsation match very well those obtained by the pertur- 
bation analysis. This oscillatory flow is characterized by propagating waves near the end walls and by steady waves in the core region of the annulus. Both solutions obtained at $R a=22,000$ are essentially two-dimensional, in agreement with experimental observations by Grigull and Hauf [11].

Acknowledgments. This work was supported in part by the IDRIS computational center. Computations on the IDRIS NEC-SX5 were made possible through grant $n^{\circ} 051265$.

\section{References}

[1] G. Petrone, E. Chénier, and G. Lauriat. Stability of free convection in air-filled horizontal annuli: Influence of the radius ratio. Int. J. Heat Mass Transfer, 47:3889-3907, 2004.

[2] Y. Tanaka, K. Iwashige, K. Fukuda, and S. Hasegawa. Three-dimensional natural convection in a inclined cylindrical annulus. Int. J. Heat Mass Transfer, $27: 747-754,1984$.

[3] Y.F. Rao, Y. Miki, K. Fukuda, Y. Takata, and S. Hasegawa. Flow patterns of natural convection in horizontal cylindrical annuli. Int. J. Heat Mass Transfer, 28:705-714, 1985.

[4] T. Fusegi and B. Farouk. A three-dimensional study of natural convection in the annulus between horizontal concentric cylinders. In Proc. 8th Int. Heat Transfer Conference, volume 4, pages 1575-1580, 1986.

[5] K. Vafai and J. Ettefagh. An investigation of transient three-dimensional buoyancy-driven flow and heat transfer in a closed horizontal annulus. Int. 
J. Heat Mass Transfer, 34(10):2555-2569, 1991.

[6] P. Cadiou. Contribution à l'étude numérique des transferts de chaleur par convection naturelle ou par convection mixte dans une cavité annulaire horizontale. PhD thesis, University of Marne-la-Vallée, 1997.

[7] M.P. Dyko, K. Vafai, and A.K. Mojtabi. A numerical and experimental investigation of stability of natural convective flows within a horizontal annulus. J. Fluid Mech., 381:27-61, 1999.

[8] M.P. Dyko and K. Vafai. Three-dimensional natural convective states in a narrow-gap horizontal annulus. J. Fluid Mech., 445:1-36, 2001.

[9] M.P. Dyko and K. Vafai. On the presence of odd transverse convective rolls in narrow-gap horizontal annuli. Phys. Fluids, 14(3):1291-1294, 2002.

[10] R.E. Powe, C.T. Carley, and E.H. Bishop. Free convective flow patterns in cylindrical annuli. Trans. ASME C: J. Heat Transfer, 91:310-314, 1969.

[11] U. Grigull and W. Hauf. Natural convection in horizontal cylindrical annuli. In Proc. of the third Int. Heat Transfer Conference, Paper No 60, volume 2, pages 182-195, 1966.

[12] J.Y. Choi and M-U Kim. Three-dimensional linear stability of natural convective flow between concentric horizontal cylinders. Int. J. Heat Mass Transfer, 36(17):4173-4180, 1993.

[13] K. Goda. A multistep technique with implicit difference schemes for calculating two- or three-dimensional cavity flows. J. Comput. Phys., 30:76-95, 1979.

[14] P. Haldenwang, G. Labrosse, S. Abboudi, and M. Deville. Chebyshev 3-d spectral and 2-d pseudospectral solvers for the Helmholtz equation. J. Comp. Phys., 55:115-128, 1984. 
[15] R.B. Wilhelmson and J.H. Ericksen. Direct solutions for Poisson's equation in three dimensions. J. Comput. Phys., 25:319-331, 1977.

[16] U. Schumann and R.A. Sweet. Fast Fourier Transform for direct solution of Poisson's equation with staggered boundary conditions. J. Comput. Phys., 75:123-137, 1988.

[17] C.J. Roy. Review of code and solution verification procedures for computational simulation. J. Comput. Phys., 205:131-156, 2005.

[18] I. Raspo, S. Hugues, E. Serre, A. Randriamampianina, and P. Bontoux. A spectral projection method for the simulation of complex three-dimensional rotating flows. Computers and Fluids, 31:745-767, 2002. 


\section{Appendix}

\section{A Numerical schemes}

\section{A.1 Helmholtz solver}

In order to exemplify the use of the Helmholtz solver, we consider the energy equation modified in terms of the temperature increment $\delta T^{n}=T^{(n+1)}-T^{(n)}$ :

$$
\begin{aligned}
& \left(1-\frac{2 \Delta t}{3 f} \widetilde{\nabla}^{2}\right) T^{(n+1)}=S_{T}^{n+1} \\
& \left(1-\frac{2 \Delta t}{3 f} \widetilde{\nabla}^{2}\right) \delta T^{(n)}=S_{T}^{n+1}-\left(1-\frac{2 \Delta t}{3 f} \widetilde{\nabla}^{2}\right) T^{(n)}
\end{aligned}
$$

where $S_{T}^{n+1}$ contains the convective terms and $\Delta t$ is the time step. By using the following second-order approximation

$$
\begin{aligned}
&\left(1-\frac{2 \Delta t}{3 f} \widetilde{\nabla}^{2}\right) \delta T^{n}=\left(1-\frac{2 \Delta t}{3}\left(\frac{1}{f} \frac{\partial}{\partial r}\left(f \frac{\partial}{\partial r}\right)+\frac{\eta^{2}}{f^{2}} \frac{\partial^{2}}{\partial \theta^{2}}+\frac{\partial^{2}}{\partial z^{2}}\right)\right) \delta T^{n} \\
&= {\left[\left(1-\frac{2 \Delta t}{3}\left(\frac{1}{f} \frac{\partial}{\partial r}\left(f \frac{\partial}{\partial r}\right)\right)\right)\left(1-\frac{2 \Delta t}{3}\left(\frac{\eta^{2}}{f^{2}} \frac{\partial^{2}}{\partial \theta^{2}}\right)\right)\right.} \\
&\left.\left(1-\frac{2 \Delta t}{3}\left(\frac{\partial^{2}}{\partial z^{2}}\right)\right)+\mathcal{O}\left(\Delta t^{2}\right)\right] \delta T^{n}
\end{aligned}
$$

accounting for that $\delta T^{n}=T^{(n+1)}-T^{(n)} \equiv \mathcal{O}(\Delta t)$ the Helmholtz equation is approximated by

$$
\begin{aligned}
\left(1-\frac{2 \Delta t}{3 f} \widetilde{\nabla}^{2}\right) \delta T^{n}= & \left(1-\frac{2 \Delta t}{3}\left(\frac{1}{f} \frac{\partial}{\partial r}\left(f \frac{\partial}{\partial r}\right)\right)\right)\left(1-\frac{2 \Delta t}{3}\left(\frac{\eta^{2}}{f^{2}} \frac{\partial^{2}}{\partial \theta^{2}}\right)\right) \\
& \left(1-\frac{2 \Delta t}{3}\left(\frac{\partial^{2}}{\partial z^{2}}\right)\right) \delta T^{n}+\mathcal{O}\left(\Delta t^{3}\right)
\end{aligned}
$$

The second order time approximation is recovered by dividing by $\Delta t$. Such a method allows the substitution of the resolution of the hepta-diagonal matrix 
by tri-diagonal matrices:

$$
\left\{\begin{array}{l}
\left(1-\frac{2 \Delta t}{3}\left(\frac{1}{f} \frac{\partial}{\partial r}\left(f \frac{\partial}{\partial r}\right)\right)\right) \delta T_{1}=S_{T}^{n+1}-\left(1-\frac{2 \Delta t}{3 f} \widetilde{\nabla}^{2}\right) T^{(n)} \\
\left(1-\frac{2 \Delta t}{3}\left(\frac{\eta^{2}}{f^{2}} \frac{\partial^{2}}{\partial \theta^{2}}\right)\right) \delta T_{2}=\delta T_{1} \\
\left(1-\frac{2 \Delta t}{3}\left(\frac{\partial^{2}}{\partial z^{2}}\right)\right) \delta T^{n}=\delta T_{2}
\end{array}\right.
$$

\section{A.2 Poisson solver}

Let $S_{i, j, k}$ the discrete right-hand side term of the Poisson equation

$$
(1 / f) \widetilde{\nabla}^{2} \phi^{(n)}=S^{(n)}
$$

and note $\mathcal{R}_{k, l}, \mathcal{A}_{k, l}$ and $\mathcal{Z}_{k, l}$ the finite volume components of the radial $(\mathcal{R})$, azimuthal $(\mathcal{A})$ and axial $(\mathcal{Z})$ matrices for the discrete approximations of the partial second-order derivatives. The Poisson equation writes :

$$
\begin{gathered}
\left(\mathcal{R}_{i, i+1} \phi_{i+1, j, k}+\mathcal{R}_{i, i} \phi_{i, j, k}+\mathcal{R}_{i, i-1} \phi_{i-1, j, k}\right)+ \\
\frac{1}{f_{i}^{2}}\left(\mathcal{A}_{j, j+1} \phi_{i, j+1, k}+\mathcal{A}_{j, j} \phi_{i, j, k}+\mathcal{A}_{j, j-1} \phi_{i, j-1, k}\right)+ \\
\left(\mathcal{Z}_{k, k+1} \phi_{i, j, k+1}+\mathcal{Z}_{k, k} \phi_{i, j, k}+\mathcal{Z}_{k, k-1} \phi_{i, j, k-1}\right)=S_{i, j, k}
\end{gathered}
$$

where $\phi_{i, j, k}$ is an approximation of $\phi\left(r_{i}, \theta_{j}, z_{k}\right)$. Using both a tensorial notation and the Einstein convention for summation between indices, we obtain:

$$
\mathcal{R}_{i, l} \phi_{l, j, k}+\delta_{i l} \frac{1}{f_{l}^{2}} \mathcal{A}_{j, m} \phi_{l, m, k}+\mathcal{Z}_{k, n} \phi_{i, j, n}=S_{i, j, k}
$$

with $\delta_{i l}$ is the Krönecker symbol. By introducing, for the azimuthal and axial coordinates, the matrices of eigenvectors $P^{\mathcal{A}}$ and $P^{\mathcal{Z}}$ and the eigenvalues $\Lambda^{\mathcal{A}}$ 
and $\Lambda^{\mathcal{Z}}$ satisfying $\mathcal{A}=P^{\mathcal{A}} \Lambda^{\mathcal{A}}\left(P^{\mathcal{A}}\right)^{-1}$ and $\mathcal{Z}=P^{\mathcal{Z}} \Lambda^{\mathcal{Z}}\left(P^{\mathcal{Z}}\right)^{-1}$ :

$\mathcal{R}_{i, l} \phi_{l, j, k}+\delta_{i l} \frac{1}{f_{l}^{2}} P_{j, p}^{\mathcal{A}} \Lambda_{p, p}^{\mathcal{A}}\left(\left(P^{\mathcal{A}}\right)^{-1}\right)_{p, m} \phi_{l, m, k}+P_{k, q}^{\mathcal{Z}} \Lambda_{q, q}^{\mathcal{Z}}\left(\left(P^{\mathcal{Z}}\right)^{-1}\right)_{q, n} \phi_{i, j, n}=S_{i, j, k}$

By multiplying the above relation by $\left(\left(P^{\mathcal{A}}\right)^{-1}\right)_{p, j}$ and $\left(\left(P^{\mathcal{Z}}\right)^{-1}\right)_{q, k}$ :

$$
\begin{aligned}
& \mathcal{R}_{i, l}\left(\left(P^{\mathcal{A}}\right)^{-1}\right)_{p, j}\left(\left(P^{\mathcal{Z}}\right)^{-1}\right)_{q, k} \phi_{l, j, k}+\frac{\delta_{i l}}{f_{l}^{2}} \Lambda_{p, p}^{\mathcal{A}}\left(\left(P^{\mathcal{A}}\right)^{-1}\right)_{p, m}\left(\left(P^{\mathcal{Z}}\right)^{-1}\right)_{q, k} \phi_{l, m, k}+ \\
& \Lambda_{q, q}^{\mathcal{Z}}\left(\left(P^{\mathcal{A}}\right)^{-1}\right)_{p, j}\left(\left(P^{\mathcal{Z}}\right)^{-1}\right)_{q, n} \phi_{i, j, n}=\left(\left(P^{\mathcal{A}}\right)^{-1}\right)_{p, j}\left(\left(P^{\mathcal{Z}}\right)^{-1}\right)_{q, k} S_{i, j, k}
\end{aligned}
$$

and using the following notations $\bar{\phi}_{l, p, q}=\left(\left(P^{\mathcal{A}}\right)^{-1}\right)_{p, j}\left(\left(P^{\mathcal{Z}}\right)^{-1}\right)_{q, k} \phi_{l, j, k}$ and $\bar{S}_{i, p, q}=\left(\left(P^{\mathcal{A}}\right)^{-1}\right)_{p, j}\left(\left(P^{\mathcal{Z}}\right)^{-1}\right)_{q, k} S_{i, j, k}$, the Poisson equation writes:

$$
\left(\mathcal{R}_{i, l}+\delta_{i l} \frac{1}{f_{l}^{2}} \Lambda_{p, p}^{\mathcal{A}}+\delta_{i l} \Lambda_{q, q}^{\mathcal{Z}}\right) \bar{\phi}_{l, p, q}=\bar{S}_{i, p, q}
$$

Thus, the calculation of $\bar{\phi}_{l, p, q}$ from $\bar{S}_{i, p, q}$ needs to solve $N_{\theta} \times N_{z}$ tri-diagonal matrices of size $N_{r}$. To improve the efficiency of the Poisson solver for regular meshes in the axial direction, Fast Fourier Transforms were used as suggested by Whilhelmson and Ericksen [15] and generalized by Schumann and Sweet $[16]$.

\section{B Space and time accuracy}

The convergence properties were checked by introducing source terms into the Navier-Stokes and energy equations [17]. The tests were performed for $R a=1000, R=3$ and $A=1$. As reported by Raspo et al. [18], the projection method, namely the Goda's method [13], may reduce the spatial accuracy of the scheme, especially near the solid walls. Thus, the second order accuracy of 
the scheme can only be rigorously checked for analytical solutions with zero normal pressure gradients at the boundaries.

\section{B.1 Spatial study}

Let $E Q(X)=0$ and $X$ the steady governing equations and the set of the unknowns, namely the velocity components, the pressure and the temperature

fields. The discrete equations are noted $\widehat{E Q}\left(\widehat{X}_{i, j, k}\right)=0$ where $\widehat{X}_{i, j, k}$ is an approximation of $X\left(r_{i}, \theta_{j}, z_{k}\right)$. Let

$$
\begin{aligned}
u(r, \theta, z)= & \sin ^{2}(\pi r) \cos (\theta) \sin ^{2}(\pi z) \\
v(r, \theta, z)= & -\sin ^{2}(\pi r) \sin (\theta) \sin ^{2}(\pi z)-\frac{f}{\eta} \pi \sin (2 \pi r) \sin (\theta) \sin ^{2}(\pi z)- \\
& \frac{f}{\eta} \pi \sin ^{2}(\pi r) \sin (\theta) \sin (2 \pi z) \\
w(r, \theta, z)= & \sin ^{2}(\pi r) \cos (\theta) \sin ^{2}(\pi z) \\
T(r, \theta, z)= & \sin (\pi r) \cos (\theta) \cos (\pi z)+\cos \left(\frac{\pi}{3}(r+1)\right) \\
p(r, \theta, z)= & \cos (\pi r) \cos (\theta) \cos (\pi z)
\end{aligned}
$$

the steady analytical field satisfying the continuity equation and all the boundary conditions. Obviously, such a field cannot satisfy the momentum and energy equations. Therefore, it is necessary to introduce source terms, which are the residuals of these two equations for the analytical solution. The difference between the analytical and numerical fields, solution of the discrete problem

$$
\widehat{E Q}\left(\widehat{X}_{i, j, k}\right)=E Q(X)\left(r_{i}, \theta_{j}, z_{k}\right)
$$

must tend toward 0 with the mesh size $h=1 / N$ :

$$
\lim _{h \rightarrow 0}\left\|\widehat{X}_{i, j, k}-X\left(r_{i}, \theta_{j}, z_{k}\right)\right\|_{\infty}=0
$$


As shown by the slopes of the curves in figure B.1, the scheme is indeed second-order accurate in space discretization.

\section{B.2 Temporal study}

The method used to check the temporal accuracy is similar to that presented in the previous section. Let

$$
\begin{aligned}
u(r, \theta, z)= & {\left[\sin ^{2}(\pi r) \cos (\theta) \sin ^{2}(\pi z)\right] \sin (\pi t) } \\
v(r, \theta, z)= & {\left[-\sin ^{2}(\pi r) \sin (\theta) \sin ^{2}(\pi z)-\frac{f}{\eta} \pi \sin (2 \pi r) \sin (\theta) \sin ^{2}(\pi z)-\right.} \\
& \left.\frac{f}{\eta} \pi \sin ^{2}(\pi r) \sin (\theta) \sin (2 \pi z)\right] \sin (\pi t) \\
w(r, \theta, z)= & {\left[\sin ^{2}(\pi r) \cos (\theta) \sin ^{2}(\pi z)\right] \sin (\pi t) } \\
T(r, \theta, z)= & {[\sin (\pi r) \cos (\theta) \cos (\pi z)] \sin (\pi t) } \\
p(r, \theta, z)= & {[\cos (\pi r) \cos (\theta) \cos (\pi z)] \sin (\pi t) }
\end{aligned}
$$

an oscillatory field satisfying the mass equation and, for sake of simplicity, homogeneous thermal boundary conditions.

If the numerical scheme is both stable and consistent then the numerical solution $\widehat{X}_{i, j, k}$ of

$$
\frac{3 \widehat{X}_{i, j, k}^{n+1}-4 \widehat{X}_{i, j, k}^{n}+\widehat{X}_{i, j, k}^{n-1}}{2 \Delta t}-\widehat{E Q}\left(\widehat{X}_{i, j, k}^{n+1}\right)=\left[\frac{d X}{d t}-\widehat{E Q}(X)\right]\left(r_{i}, \theta_{j}, z_{k},(n+1) \Delta t\right)
$$

must converge toward the analytical field:

$$
\lim _{\Delta t \rightarrow 0}\left\{\max _{n}\left\|\widehat{X}_{i, j, k}^{n+1}-X\left(r_{i}, \theta_{j}, z_{k},(n+1) \Delta t\right)\right\|_{\infty}\right\}=0
$$


Remark that the discrete expression of the volumetric source term is used to limit the effect of the finite space accuracy on the temporal convergence.

As illustrated in figure B.2, the time accuracy of the scheme is of second order. It is worth noticing that slopes of the curves decrease for the smallest time steps due to the time splitting method that produces the so-called numerical boundary layer. 


\section{List of Figures}

1 Geometry.

2 Isotherms and velocity vectors in the vertical $(r, z)$ top section, for $R=1.6$ and $A=6.66$; (a) $R a=1000$ and (b) $R a=2000$.

3 Isotherms and velocity vectors in the vertical $(r, z)$ top section, $R=2.2, A=8.33$; (a) $R a=10^{3}$, (b) $R a=4 \cdot 10^{3}$ and (c) $R a=10^{4}$.

4 Two streamlines for $R=1.7, A=6$ and $R a=6 \cdot 10^{3}$.

5 Isotherms and velocity vectors in the vertical $(r, z)$ top section for $R=1.7, A=6$ and $R a=6 \cdot 10^{3}$.

6 Isotherms and velocity vectors in the vertical $(r, z)$ top section achieved by two initial conditions, for $R=1.7, A=6$ and $R a=10^{4}$; (a) Fluid flow initialized by rest conditions and (b) Fluid flow initialized by the $3 D$-flow at $R a=6 \cdot 10^{3}$.

$7 \quad$ Isosurfaces of the axial component of the velocity and streamlines for $R=1.7, A=20$ and $R a=22,000$.

8 Axial velocity distribution in the vertical $(r, z)$ top section for $R=1.7, A=20$ and $R a=22,000$.

9 Spatio-temporal diagram for $R=1.7, A=20$ and $R a=22,000$.

B.1 Spatial convergence analysis, $\Delta X=\mid \widehat{X}_{i, j, k}-X\left(r_{i}, \theta_{j}, z_{k}\right) \|_{\infty}$. 
B.2 Temporal convergence analysis, $\max _{n}\left\|\widehat{X}_{i, j, k}^{n+1}-X\left(r_{i}, \theta_{j}, z_{k},(n+1) \Delta t\right)\right\|_{\infty}$. 


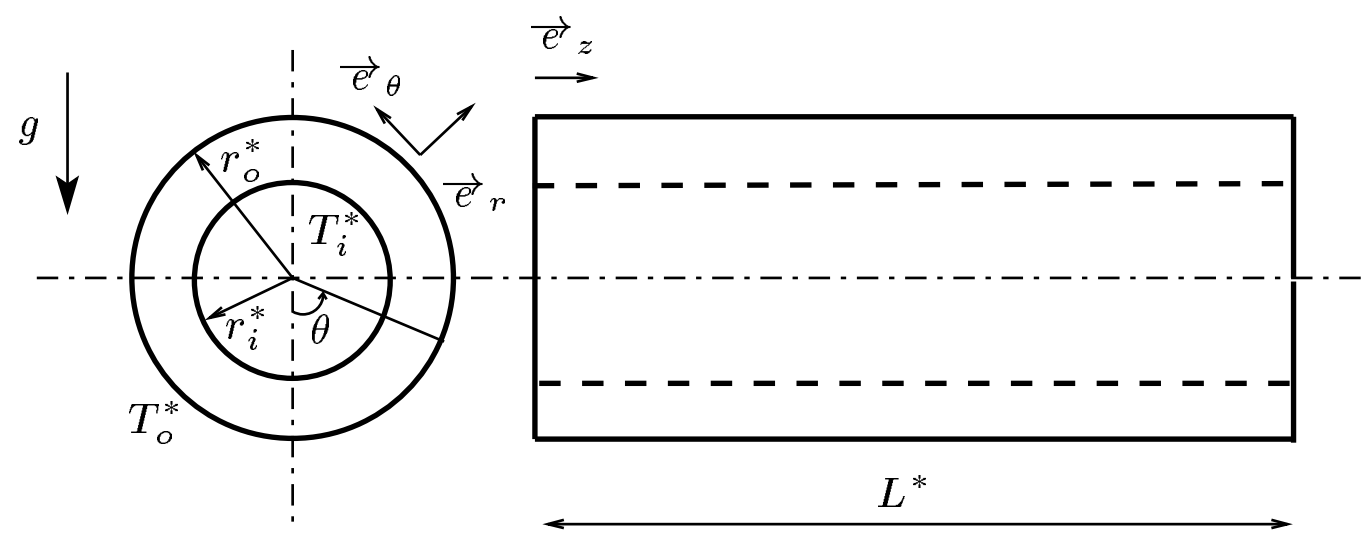

Fig. 1. Geometry.

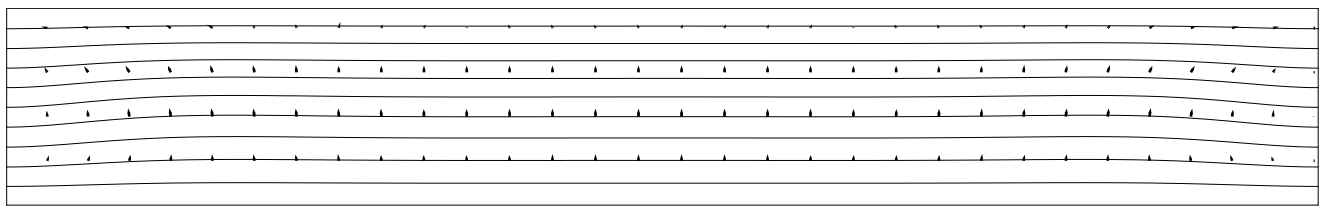

(a) $R a=1000$

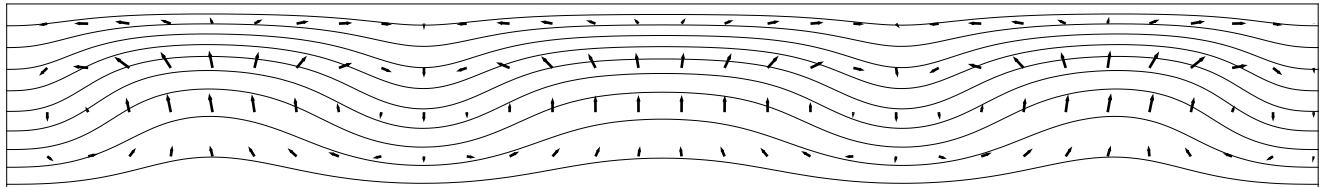

(b) $R a=2000$

Fig. 2. Isotherms and velocity vectors in the vertical $(r, z)$ top section, for $R=1.6$ and $A=6.66$. 


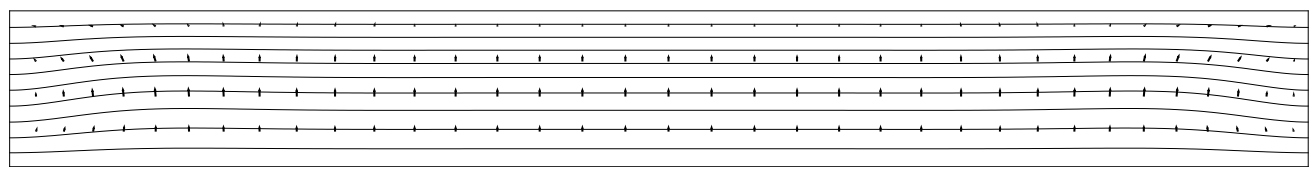

(a) $R a=10^{3}$

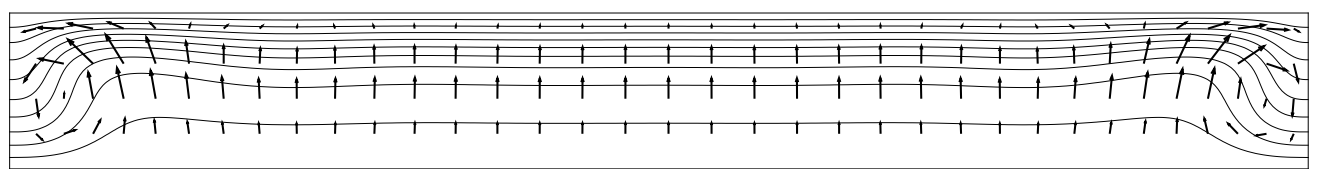

(b) $R a=4 \cdot 10^{3}$

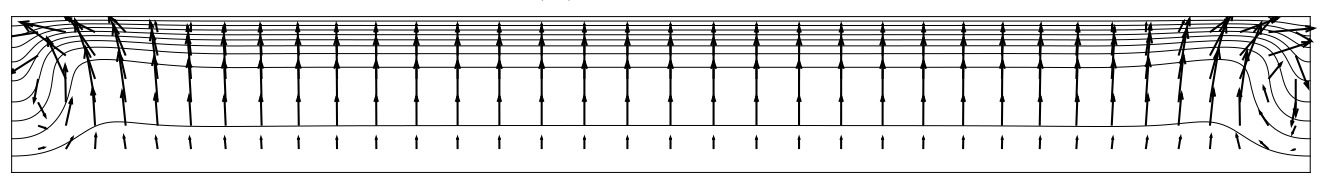

(c) $R a=10^{4}$

Fig. 3. Isotherms and velocity vectors in the vertical $(r, z)$ top section, $R=2.2$, $A=8.33$. 


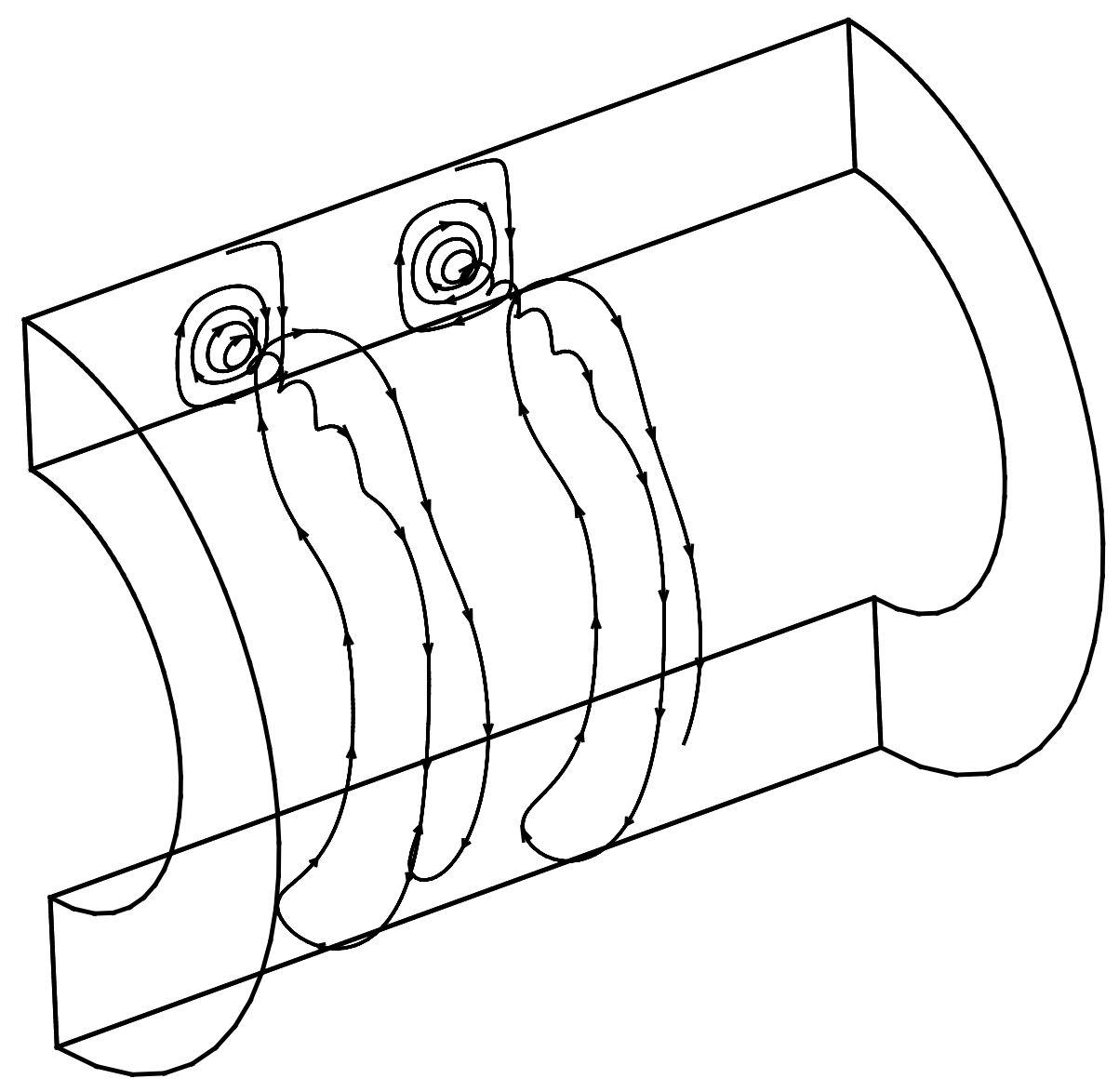

Fig. 4. Two streamlines for $R=1.7, A=6$ and $R a=6 \cdot 10^{3}$.

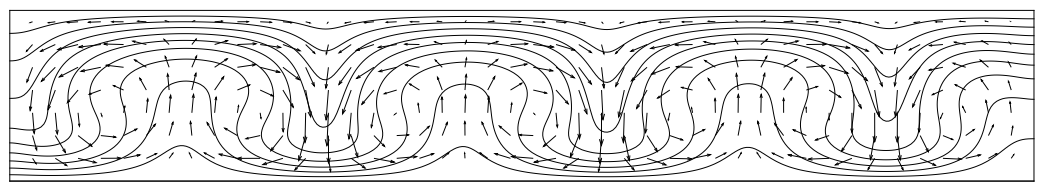

Fig. 5. Isotherms and velocity vectors in the vertical $(r, z)$ top section for $R=1.7$, $A=6$ and $R a=6 \cdot 10^{3}$. 


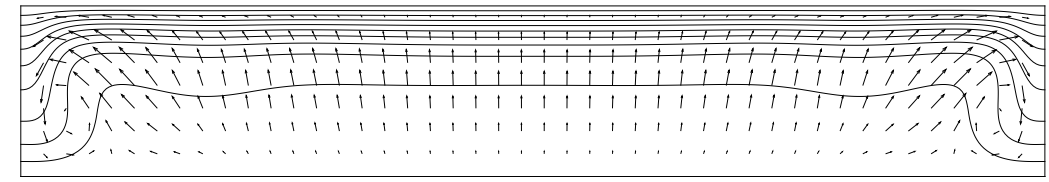

(a) Fluid flow initialized by rest conditions.

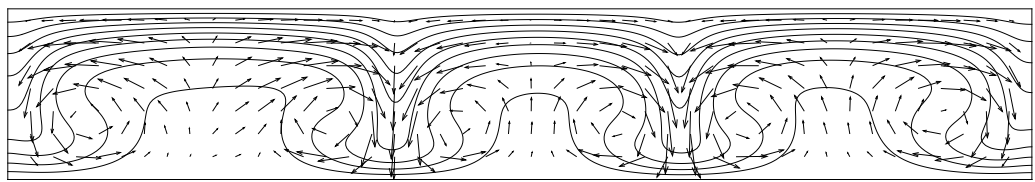

(b) Fluid flow initialized by the $3 D$-flow at $R a=6 \cdot 10^{3}$.

Fig. 6. Isotherms and velocity vectors in the vertical $(r, z)$ top section achieved by two initial conditions, for $R=1.7, A=6$ and $R a=10^{4}$.

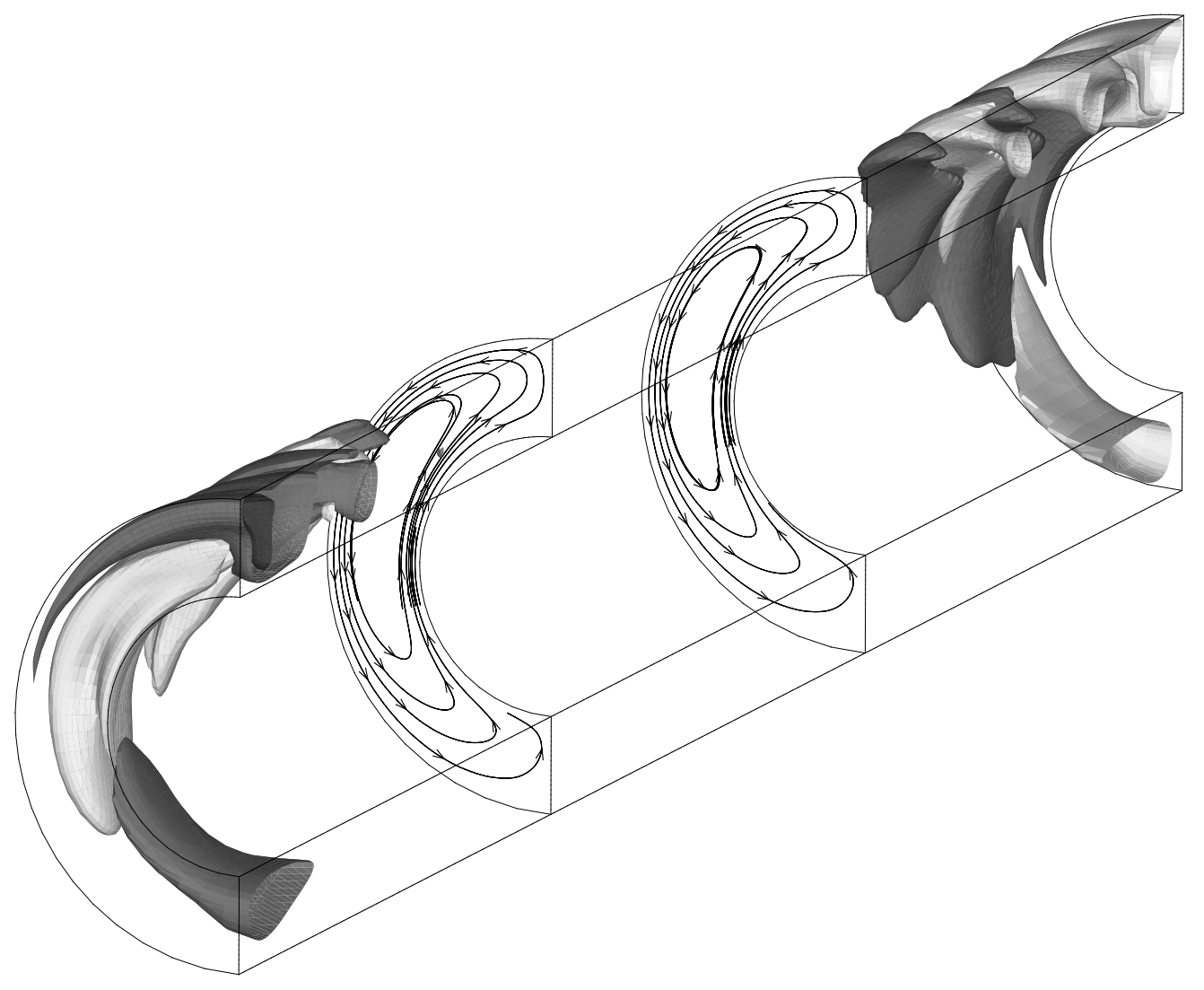

Fig. 7. Isosurfaces of the axial component of the velocity and streamlines for $R=1.7$, $A=20$ and $R a=22,000$. 


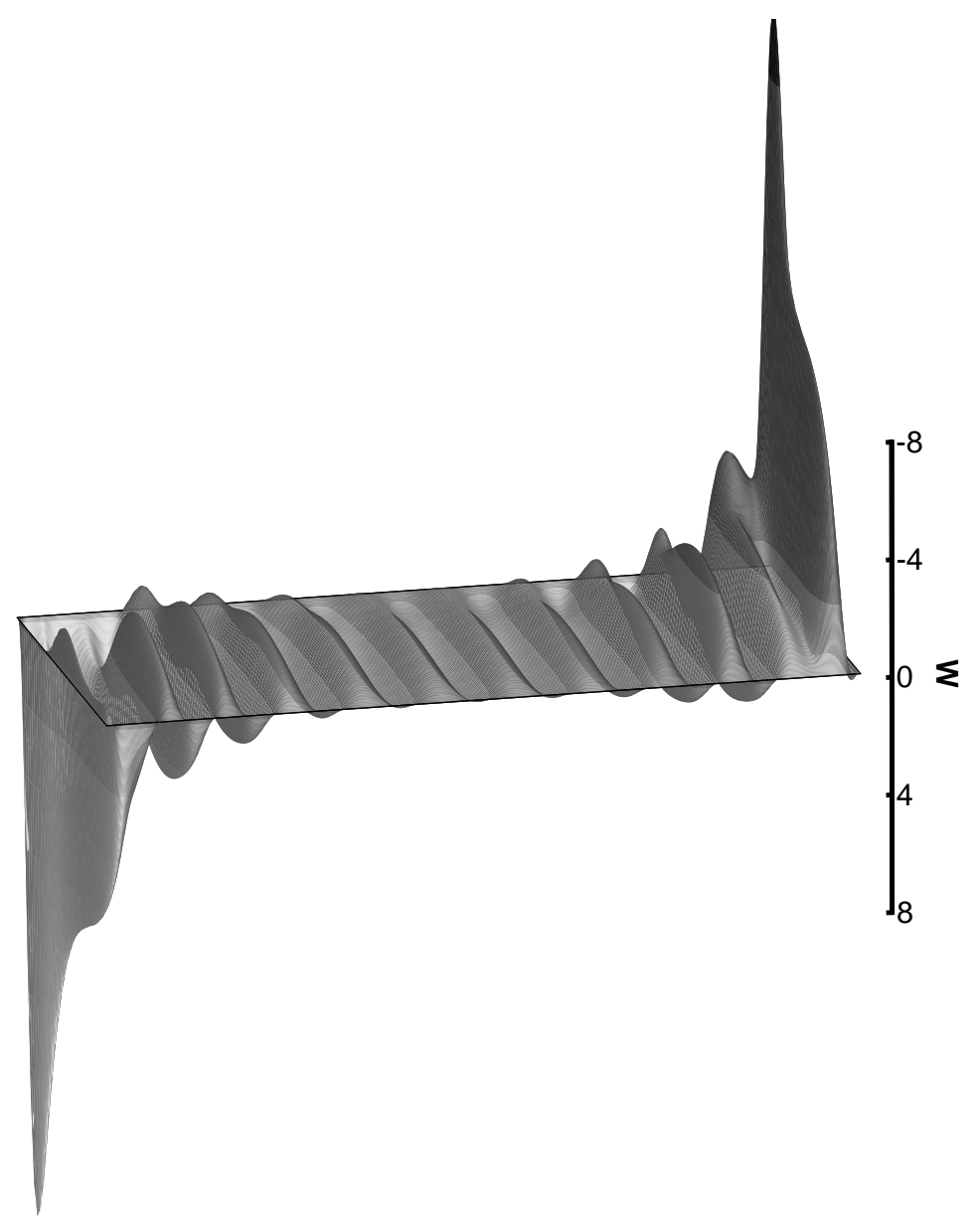

Fig. 8. Axial velocity distribution in the vertical $(r, z)$ top section for $R=1.7$, $A=20$ and $R a=22,000$. 


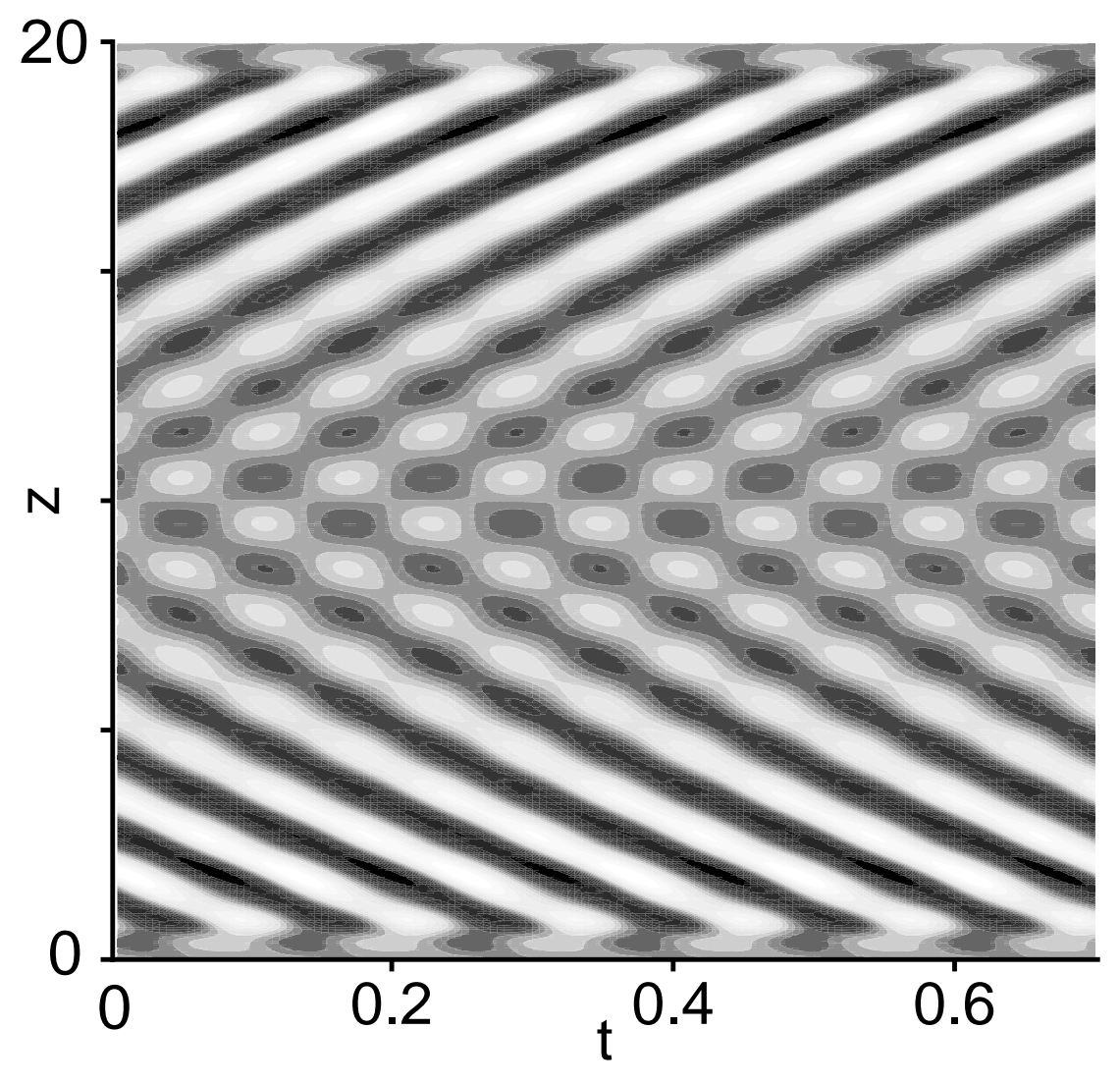

Fig. 9. Spatio-temporal diagram for $R=1.7, A=20$ and $R a=22,000$. 


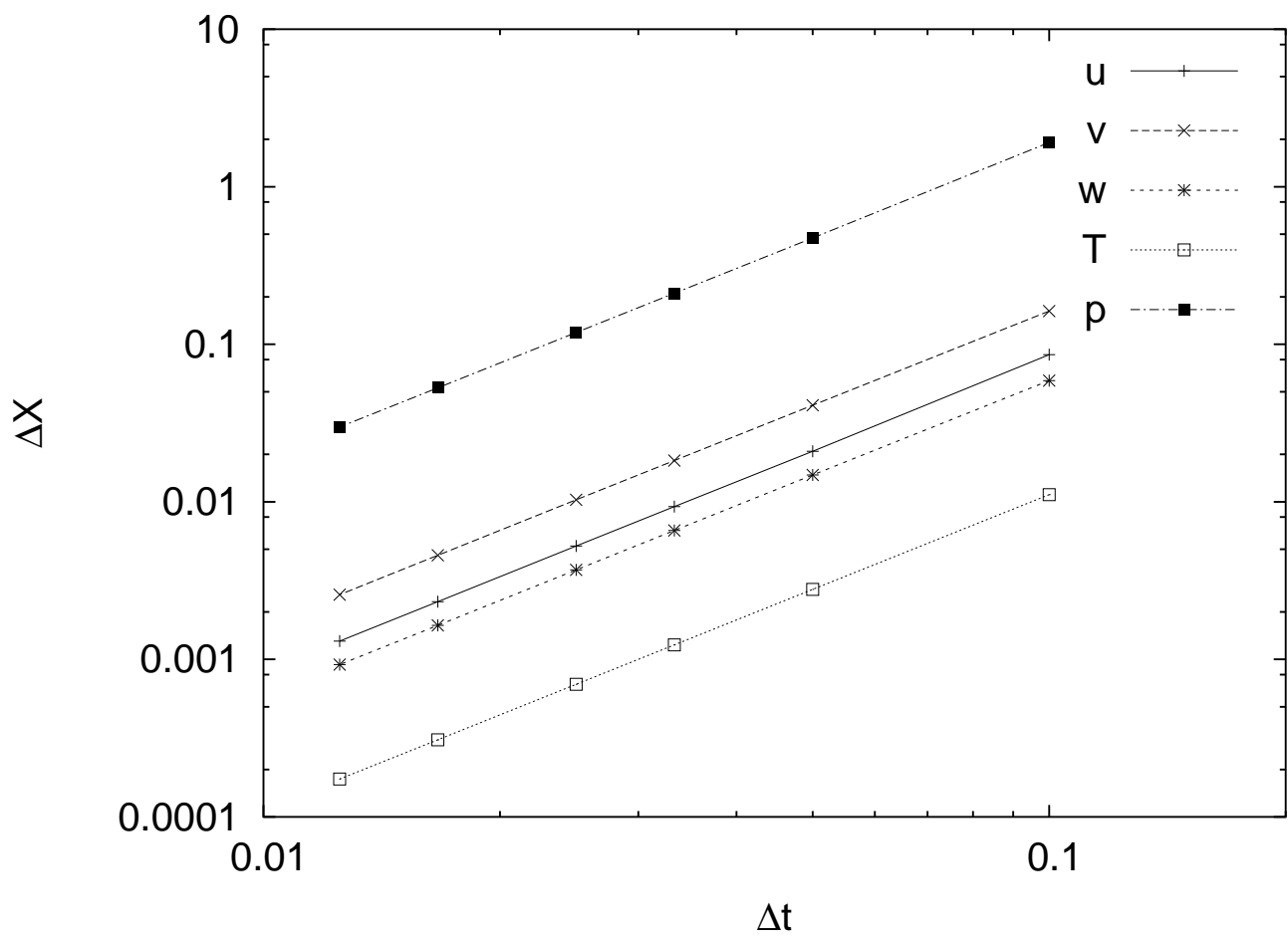

Fig. B.1. Spatial convergence analysis, $\Delta X=\mid \widehat{X}_{i, j, k}-X\left(r_{i}, \theta_{j}, z_{k}\right) \|_{\infty}$. 


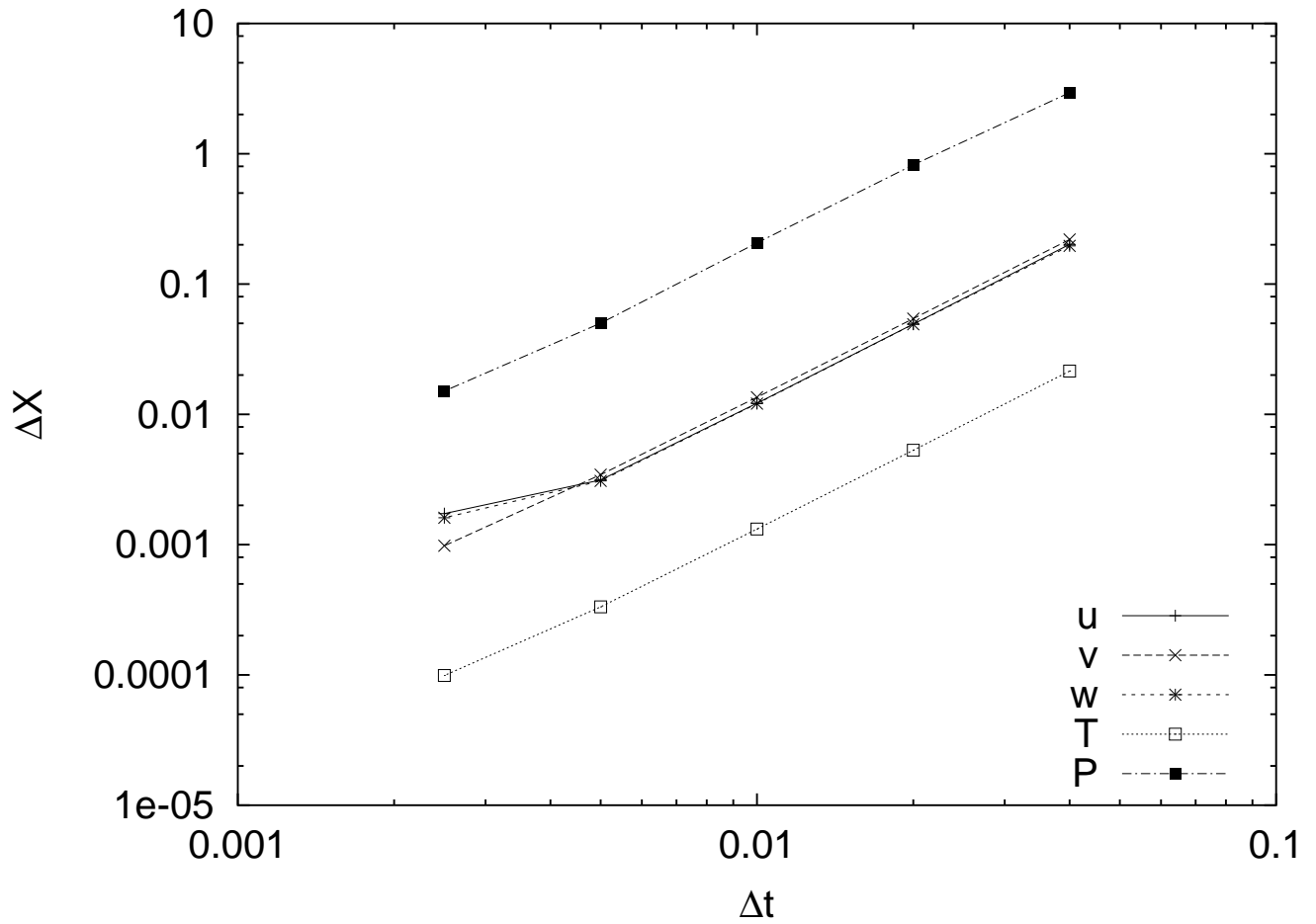

Fig. B.2. Temporal convergence analysis, $\max _{n}\left\|\widehat{X}_{i, j, k}^{n+1}-X\left(r_{i}, \theta_{j}, z_{k},(n+1) \Delta t\right)\right\|_{\infty}$. 